Research Article

\title{
Suppression of Supersonic Cavity Oscillations Using Pulsed Upstream Mass Injection
}

\author{
Weipeng Li \\ Department of Aeronautics and Astronautics, Shanghai Jiaotong University, Shanghai 200-240, China \\ Correspondence should be addressed to Weipeng Li; liweipeng@sjtu.edu.cn
}

Received 3 May 2016; Accepted 7 September 2016

Academic Editor: Keh-Chin Chang

Copyright (C) 2016 Weipeng Li. This is an open access article distributed under the Creative Commons Attribution License, which permits unrestricted use, distribution, and reproduction in any medium, provided the original work is properly cited.

Pulsed upstream mass injection is examined to suppress supersonic cavity oscillations. The efficiency and physics of the noise control are investigated by large-eddy simulations of a turbulent flow $\left(M_{\infty}=2.0, \operatorname{Re}_{D}=10^{5}\right)$ past a rectangular cavity with a length-to-depth ratio of 2 . Results show that the pulsed mass injection behaves less effectively in reducing the cavity oscillations than the steady one. The primary reason is that the pulsed mass injection is ineffective in lifting up the cavity shear layer and in suppressing the turbulent fluctuations in the shear layer. It concluded that breakup of the large-scale vorticial structures into a smaller length scale reveals direct links existing between the large-scale vortices and the radiation of the cavity resonances.

\section{Introduction}

One of the most remarkable features in supersonic flow past an open cavity is the existence of self-sustained flow oscillations, which is derived by a fluid-acoustic feedback mechanism and results in intense resonant noise. The radiation of the resonant noise may impact nearby equipment and environment harmfully, causing, for example, structural vibrations and fatigue, adverse effects on internal weapons' carriages, and undesirable noise [1-6]. To date, the development of an effective and stable control method for supersonic cavity oscillations is still a challenging work [7]. Reviews of cavity noise control have been given by Colonius [3], Rowley and Williams [4], and Cattafesta et al. [5]. The key strategy in controlling of the cavity oscillations is to disrupt the feedback mechanism [1] by inducing or forcing disturbances with additional devices or modification of cavity geometries. Upstream mass injection is an attractive control method [813]. A brief review has been given in our previous work [14], and two primary mechanisms have been demonstrated to be directly responsible for the noise suppression using steady upstream mass injection: lifting up of the cavity shear layer and damping of the shear layer instability. Here, we attempt to suppress the supersonic cavity oscillations with pulsed upstream mass injection.
The development of large-scale vortices in the cavity shear layer had been demonstrated to be a key factor for the generation of acoustic waves near the cavity trailing edge, which propagate upstream and excite the shear layer as a feedback [15]. Figure 1 shows a schematic of the noise control strategy using pulsed mass injection. If the upstream mass injection operates at a frequency different from the frequency of the dominant resonance (which has the maximum sound pressure level), the large-scale vortices in the cavity shear layer could be broken up into a smaller length scale. The breakup of the large-scale vortices into a smaller length scale is expected to redistribute the energy of the vortexwall interaction and alleviate pressure imprints on the cavity downstream wall. Rizzetta and Visbal [10] applied pulsed mass injection at a very high frequency to suppress the resonances and oscillatory modes in supersonic cavity. Results showed that the mass injection altered characteristics of the vortices forming and decreased the amplitude of shear layer excursions in the vertical direction. Few studies have been conducted to disturb the scales of the large-scale vortices and to analyze the relationship between the wavelength of the large-scale vortices and the frequency of the pulsed mass injection.

This study aims to clarify the efficiency and physics of the "vortex-breakup" approach for the suppression of the 


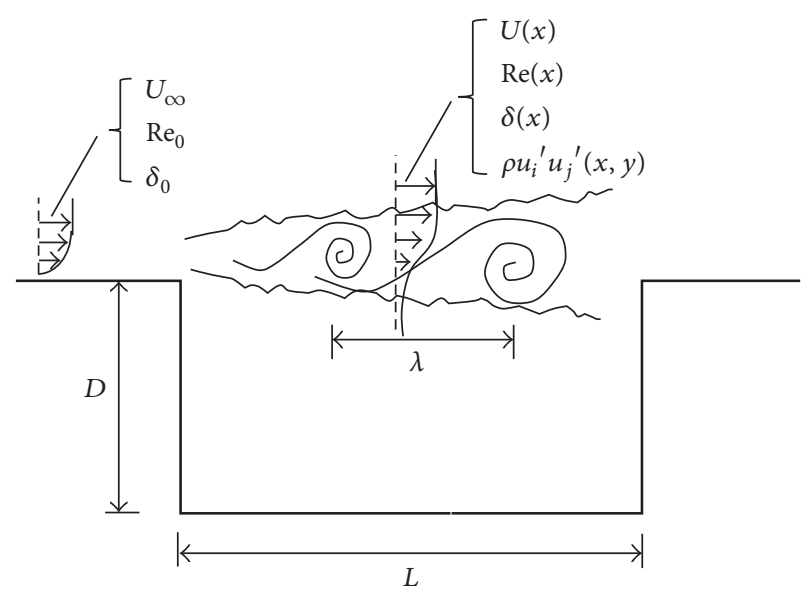

(a) Baseline

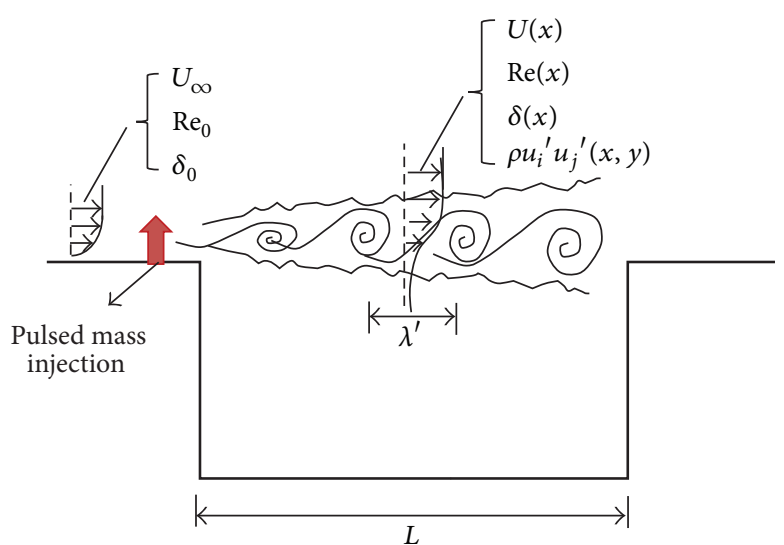

(b) With pulsed mass injection

FIGURE 1: Schematic of the noise control strategy using pulsed mass injection.

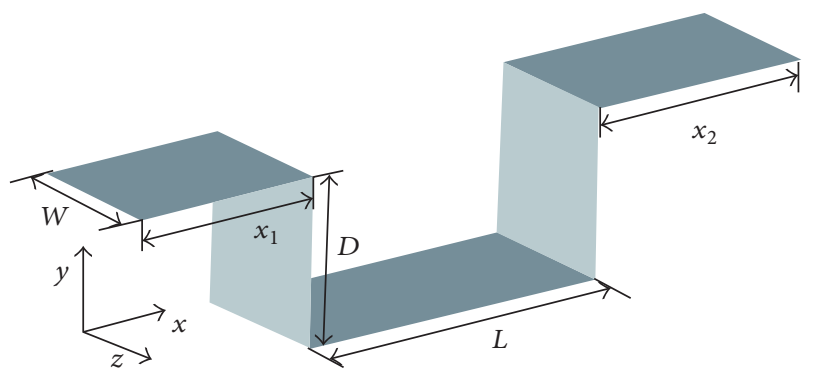

FIGURE 2: Schematic of the cavity geometry.

supersonic cavity oscillations. Large-eddy simulations are performed. Since it is a supplement of our previous study [14], brief introductions of problem setting and numerical methods are given in Sections 2 and 3, respectively. In Section 4, we will discuss the key flow and noise features of the simulation results. Conclusions are made in Section 5.

\section{Problem Setting}

A turbulent gas flow past a rectangular cavity of $L / D=2$ is simulated, where $L$ and $D$ are the length and depth of the cavity, respectively. It belongs to open cavities for which typically $1 \leq L / D \leq 10$ [6]. Figure 2 shows a schematic of the cavity geometry. A distance of $x_{1}=D$ and $x_{2}=1.5 \mathrm{D}$ from the cavity lips is extended in the upstream and downstream direction, respectively. No sidewall is defined in this cavity model. The width of the cavity $W$ is $0.9 D$.

A slotted gas jet is placed upstream of the cavity leading edge. Figure 3 shows the geometry of the slotted jet (out of scale). The jet spans the computational domain and has a streamwise length of $d x_{j 1}=0.08 D$. It is positioned at a distance of $d x_{j 2}=0.08 D$ from the leading-edge cavity lip.

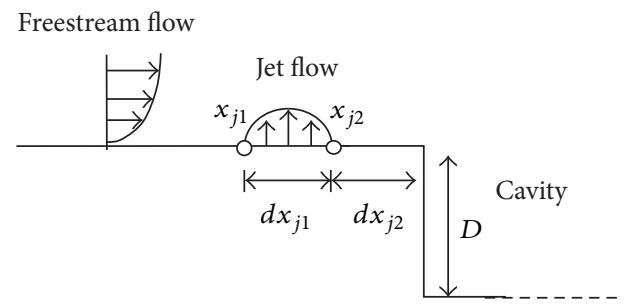

FiguRE 3: Geometry of the slotted jet (out of scale).

The mass injection is simulated by specifying a jet, which is ejected vertically through the slot. Its velocity profile is defined by the following [10]:

$$
v_{\text {jet }}=A \times a_{\infty} \times \sin \left[\pi\left(\frac{x-x_{j 1}}{x_{j 2}-x_{j 1}}\right)\right] \times \cos ^{2}(\pi F \times t),
$$

where $A$ represents the maximum Mach number of the jet flow, $a_{\infty}$ is the sound speed of the freestream flow, $x_{j 1}$ and $x_{j 2}$ are the $x$-coordinates of the slot edges, and $F$ is the operation frequency of the jet. At the plane of the jet exit, the pressure is obtained from the inviscid normal momentum equation, and the jet is assumed to be isothermal at the wall temperature.

Four simulation cases are performed. The flow conditions are listed in Table 1. We set the freestream Mach number $M_{\infty}=2.0$ and chose a moderate Reynolds number of $\operatorname{Re}_{D}=$ $10^{5}$. The inflow is turbulent, and the boundary-layer thickness $\delta_{0}(99 \%$ velocity thickness) is fixed to be $0.1 D$. The first noise control case (Steady) is of a steady jet with $A=0.4$. Two more cases with pulsed mass injection (Pulsed-F2 and Pulsed-F10) are calculated. The pulsing frequency varies from $2 f_{d}$ to $10 f_{d}$, where $f_{d}$ is the frequency of the dominant resonance (with the maximum sound pressure level) in the Baseline case. $f_{d}$ is corresponding to the wavelength $(\lambda)$ of the most energetic large-scale vortices in the cavity shear layer [14]. Thus the Pulsed-F2 case is set to break up the length scale of the most energetic large-scale vortices to a smaller one $\left(\lambda^{\prime}=\lambda / 2\right)$, and 
TABLE 1: Flow conditions of the cavity noise control with/without upstream mass injection.

\begin{tabular}{lcccccccc}
\hline Cases & $M$ & $\mathrm{Re}_{D}$ & $L / D$ & $W / D$ & $\delta_{0} / D$ & $A$ & $F$ & $C_{\mu}$ \\
\hline Baseline & 2.0 & $10^{5}$ & 2.0 & 0.9 & 0.1 & 0.0 & 0.0 & 0.0 \\
Steady & 2.0 & $10^{5}$ & 2.0 & 0.9 & 0.1 & 0.4 & 0.0 & 0.026 \\
Pulsed-F2 & 2.0 & $10^{5}$ & 2.0 & 0.9 & 0.1 & 0.4 & $2 f_{d}$ \\
Pulsed-F10 & 2.0 & $10^{5}$ & 2.0 & 0.9 & 0.1 & 0.4 & 0.0025 \\
\hline
\end{tabular}

it is also termed as the "vortex-breakup" case. The Pulsed-F10 case, which will introduce high-frequency mass forcing with respect to the dominant cavity oscillatory mode, is chosen for a comparison with the "cortex-breakup" case. The massflow rate of the injection which is expressed in terms of a momentum flux coefficient $C_{\mu}[16]$ is shown in Table 1.

\section{Numerical Methods}

The governing equations are three-dimensional compressible Navier-Stokes equations in conservative form. Implicit large-eddy simulations are performed. Structured grids are adopted, and the total number of grid points is 11.4 million. Details of the numerical methods and validations could be found in our previous works $[14,15,17]$.

\section{Results and Discussion}

The most interesting point is to discuss the efficiency of noise suppression with the mass injection. Figure 4 shows the pressure spectra at the midpoint of the cavity downstream wall. The sound pressure level (SPL) is defined by SPL = $20 \log _{10}\left(p / p_{\text {ref }}\right)$, where $p_{\text {ref }}=2.0 \times 10^{-5}[\mathrm{~Pa}] / 101300[\mathrm{~Pa}] \times$ $p_{\infty}$. The standard Strouhal number St is defined by St $=$ $f L / U_{\infty}$, where $f$ is the frequency, $L$ is the length of the cavity, and $U_{\infty}$ is the freestream velocity. Noise reductions of up to $15 \mathrm{~dB}$ in the cavity dominant resonance $(\mathrm{St} \approx 1.2)$ and $4 \mathrm{~dB}$ in the broadband noise are obtained for the case with steady mass injection. However, for the cases with pulsed mass injection the noise attenuation is less effective. The broadband noise for the Plused-F10 case is reduced by approximately $2 \mathrm{~dB}$, but no notable attenuation is obtained for the Plused-F2 cases. An extra strong resonance is observed in the Pulsed-F2 case at a frequency of $S t \approx 2.4$ that is the same as the pulsing frequency of the slotted jet. Figure 5 shows the distributions of the root-mean-square of pressure fluctuations $p_{\text {rms }}$ along the internal cavity walls, including the front wall, the bottom wall, and the rear wall. It represents the distributions of overall sound pressure levels (OASPL). All the control cases are able to suppress the dynamic pressure loads on the cavity walls and the steady mass injection results in more significant attenuation than that of the pulsed mass injection.

In order to examine the longitudinal size of the large-scale vortices in the cavity shear layer, we define a parameter $W^{*}$, which represents the vorticial coherence in the shear layer, averaged laterally at the mid of the cavity $(x=0.5 L)$,

$$
W^{*}=\int_{y=-0.25 D}^{y=0.25 D}|\omega|_{x=0.5 L} d y .
$$

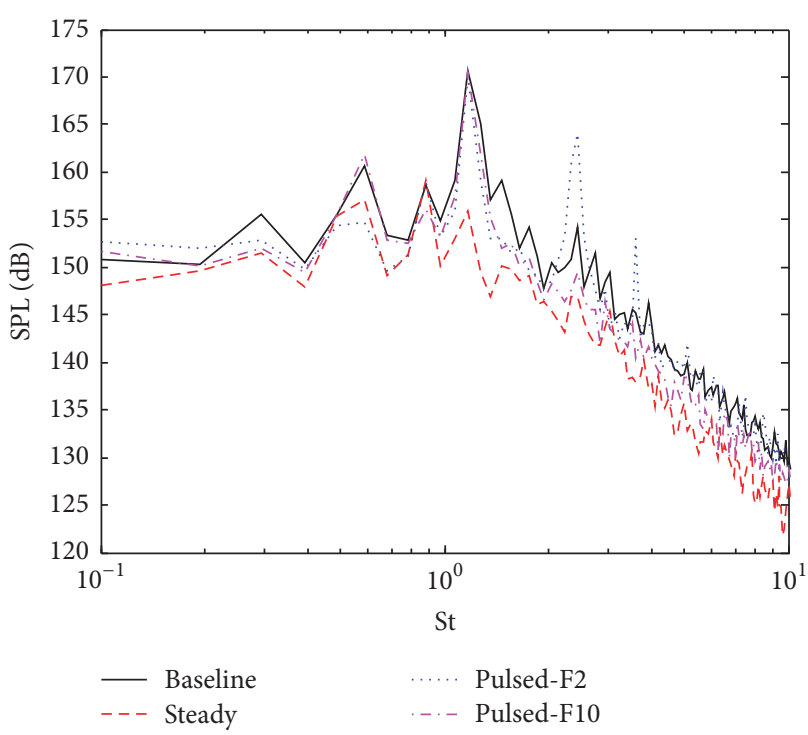

FIGURE 4: Pressure spectra at the midpoint of cavity downstream wall.

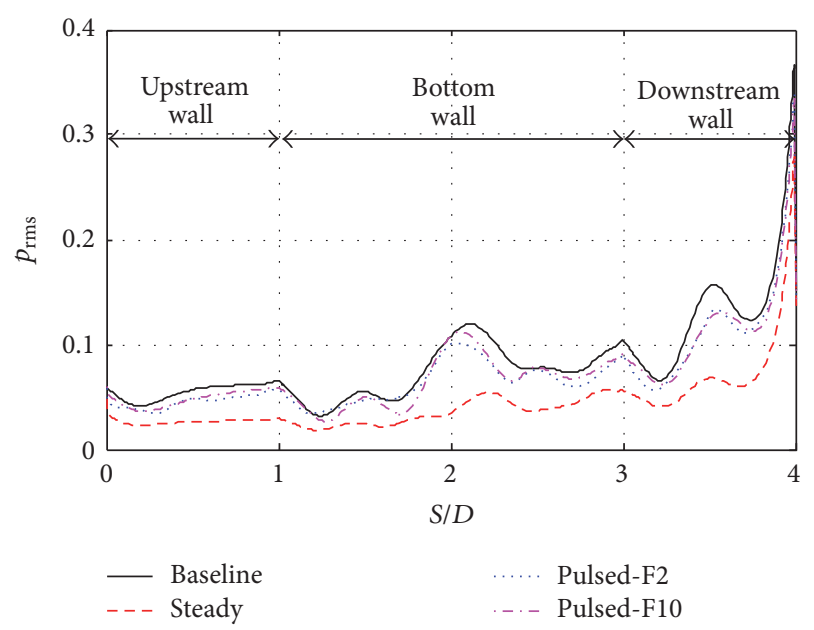

FIGURE 5: Distribution of root-mean-square of pressure fluctuations $\left(p_{\text {rms }}\right)$ along internal cavity walls.

Here $|\omega|_{x=0.5 L}$ denotes the absolute vorticity in the flow, averaged spanwise at $x=0.5 \mathrm{~L}$. An autocorrelation analysis of $W^{*}$ is performed. Figure 6 shows the autocorrelation coefficients of $W^{*}$. The longitudinal size of vortices in the cavity shear layer, or the wavelength of the shear layer 


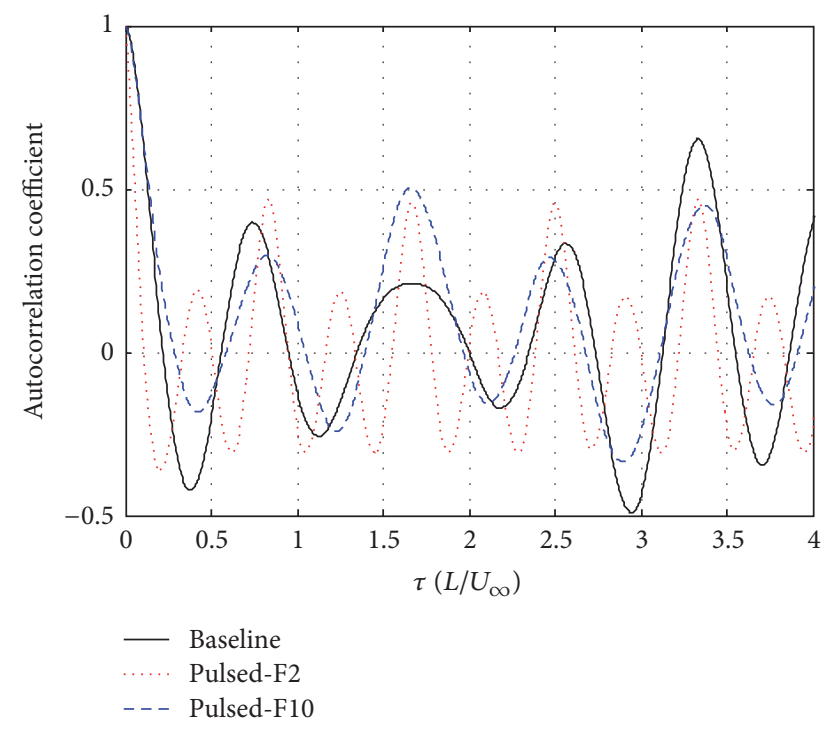

FIgURE 6: Normalized autocorrelation of $W^{*}$ at the streamwise midpoint of the cavity shear layer.

instability, can be estimated from the time lag $\tau$ (normalized by $L / U_{\infty}$ ) between two peaks. Figure 6 suggests that the mass injection in the Pulsed-F2 case does introduce an action to break up the large-scale vorticial structures into a smaller length scale, approximately half of that in the Baseline case. The extra resonance $(\mathrm{St} \approx 2.4)$ at the frequency two times larger than the dominant oscillatory mode is just corresponding to the frequency of the jet excitation and the wavelength of the large-scale vortices in the cavity shear layer. Despite the fact that the "vortex-breakup" approach may lead to an extra undesirable resonance, it provides evidence that the developing of the large-scale vortices in the cavity shear layer is directly linked to the radiation of the resonances. For the Pulsed-F10 case the pulsed mass injection does not affect the wavelength of the large-scale vortices. It indicates that the high-frequency disturbances caused by the injection of the Pulsed-F10 case may dissipate quickly and yield to the original dynamic oscillatory modes existing in the Baseline case. The result of the Steady case could be found in our previous work [14].

Due to the interaction between the incoming attached flow and the vertical slotted jet, the cavity shear layer is expected to be displaced upwards for the noise control cases. The central spatial positions of the shear layer are extracted from the time-averaged flow fields where $u=0.5 U_{\infty}$, and it is plotted in Figure 7. The position where $x / D=0$, $y / D=0$ is the cavity leading-edge lip. These curves represent the center of the cavity shear layer roughly, since the mean velocity profile of the cavity shear layer slightly depart from the self-similarity rule which is usually observed in free shear layers and mixing layers [18]. The plots in Figure 7 illustrates that the cavity shear layer is lift up over the whole length of the cavity for the three control cases considered. The lifting up of the cavity shear layer is able to alleviate the shear layer impingement on the cavity aft wall, and as a response the strength of the noise radiation is reduced. The plots in

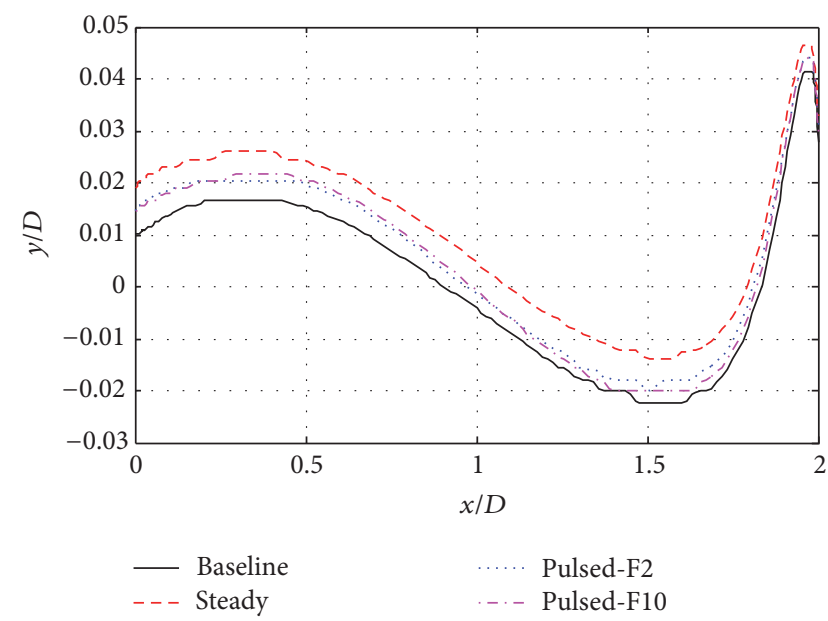

FIGURE 7: Spatial positions of $u=0.5 U_{\infty}$ in the cavity shear layer.

Figures 7 and 4 also suggest that higher amplitude "lifting up" of the cavity shear layer in the Steady case results in more noise reduction achieved. Figure 8 shows contours of rootmean-square values of the vertical velocity fluctuations $\left(v_{\text {rms }}\right)$. The steady mass injection could significantly suppress the strength of $v_{\text {rms }}$ both in the shear layer and in the vicinity of the cavity aft wall. For the pulsed injection cases the reduction of $v_{\text {rms }}$ is observed in smaller amplitude and the reduction of the shear layer excursions in the vertical direction is not notable. The reason for this is the fact that the pulsed mass injection has less capability to alleviate the impingement of the shear layer on the cavity aft wall than the steady one.

Figure 9 shows the instantaneous relations between the vertical velocity fluctuations $\left(v^{\prime}\right)$ and the pressure fluctuations $\left(p^{\prime}\right)$ in the cavity shear layer at $x=0.5 \mathrm{~L}, u=0.5 U_{\infty}$. This position is selected since it is far from the leading and trailing edge of the cavity, and here the influence of the upstream turbulent inflow and the impingement downstream is minimized as much as possible. The plots in Figure 9 suggest that the steady mass injection behaves more effective in controlling the turbulent fluctuations in the cavity shear layer. For the two cases with the pulsed mass injection, the higher-frequency one (Pulsed-F10) works better than the "vortex-breakup" one (Pulsed-F2). The different features of the turbulent fluctuations are considered as another reason related to the efficiency of the noise suppression between the pulsed and steady mass injection.

\section{Conclusion}

Large-eddy simulations of a turbulent flow $\left(M_{\infty}=2.0, \operatorname{Re}_{D}\right.$ $=10^{5}$ ) past a rectangular cavity with a length-to-depth ratio of 2 are performed to clarify the efficiency and physics of supersonic noise suppression using pulsed mass injection. A "vortex-breakup" approach is proposed and tested. It is based on the assumption that the breakup of the largescale vortices into a smaller length scale is expected to redistribute the energy of the vortex-wall interaction and alleviate pressure imprints on the cavity downstream wall. 


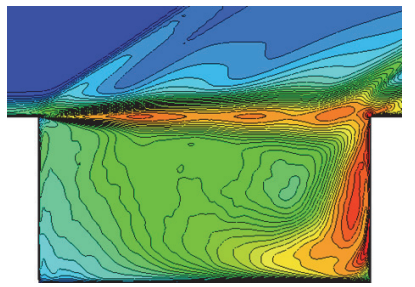

(a) Baseline

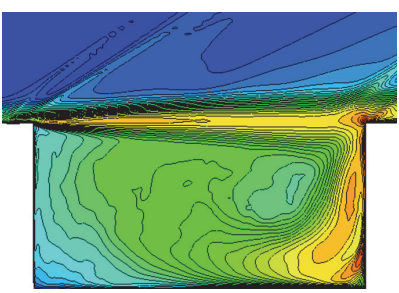

(b) Steady

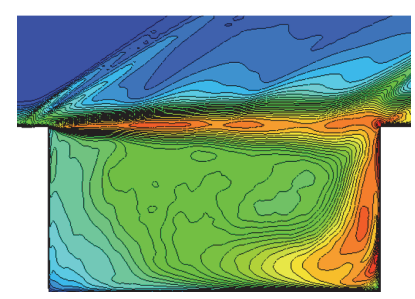

(c) Pulsed-F2

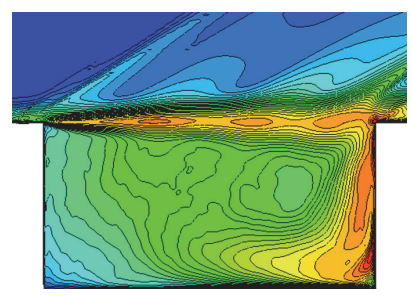

(d) Pulsed-F10

FIGURE 8: Contours of root-mean-square values of the vertical velocity fluctuations $\left(0<v_{\mathrm{rms}}<0.27 U_{\infty}\right.$, from blue to red).

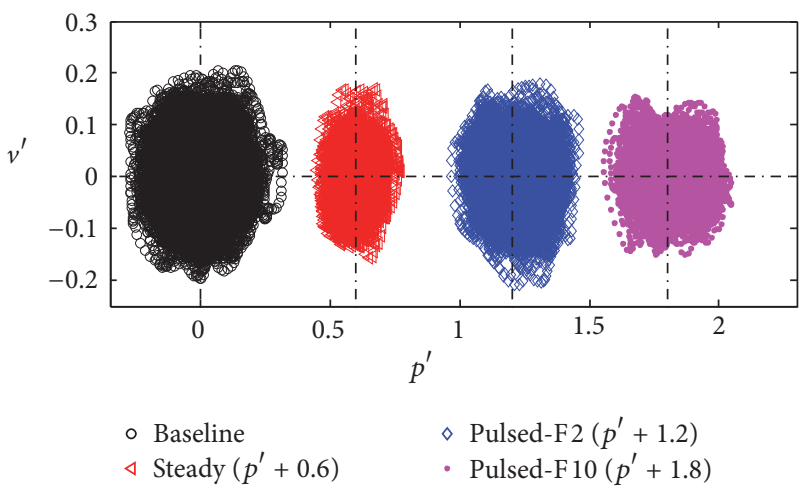

FIGURE 9: Instantaneous relations between the vertical velocity fluctuations $\left(v^{\prime}\right)$ and the pressure fluctuations $\left(p^{\prime}\right)$ in the shear layer where $x=0.5 L, u=0.5 U_{\infty}$.

Although the "vortex-breakup" approach is demonstrated to be not effective in controlling of the supersonic cavity oscillations and even an extra strong resonance may be induced, valuable conclusions on the controlling and the mechanism of the cavity oscillations need to be stated.

First, the pulsed mass injection is less effective in reducing the cavity oscillations than the steady one with the same injection amplitude. The primary reason is that the pulsed mass injection behaves ineffectively to lift up the cavity shear layer and to suppress the turbulent fluctuations in the shear layer. The reduction of the shear layer excursions in the vertical direction is not notable for cases with pulsed mass injection either. Secondly, breakup of the large-scale vorticial structures into a smaller length scale leads to an extra intense resonance, revealing direct links between the large-scale vortices and the radiation of the cavity resonances. When the pulsing frequency exceed a certain value, the dynamic oscillatory modes are similar to that of the Baseline case. Investigating the critical pulsing frequency is of interest in the future work. Regarding suppressing the forming and the developing of the large-scale vortices in the cavity shear layer, it is recommended to perform out-of-phase pulsed mass injection or feedback-loop control with reduced-order modeling.

\section{Competing Interests}

The author declares that they have no competing interests.

\section{Acknowledgments}

The author acknowledges the support of National Natural Science Foundation of China (no. 11202131), National Basic Research Program of China (973 Program), grant no. 2014CB744804, and Shanghai Key Lab of Vehicle Aerodynamic and Vehicle Thermal Management Systems.

\section{References}

[1] J. E. Rossiter, "Wind tunnel experiments on the flow over rectangular cavities at subsonic and transonic speed," Aeronautical Research Council Reports and Memoranda 3438, 1964.

[2] D. Rockwell and E. Naudascher, "Review—self-sustaining oscillations of flow past cavities," Transactions of the ASME, Journal of Fluids Engineering, vol. 100, no. 2, pp. 152-165, 1978.

[3] T. Colonius, "An overview of simulation, modeling, and active control of flow/acoustic resonance in open cavities," AIAA Paper 2001-76, 2001.

[4] C. W. Rowley and R. D. Williams, "Dynamics and control of high-reynolds-number flow over open cavities," Annual Review of Fluid Mechanics, vol. 38, pp. 251-276, 2006.

[5] L. N. Cattafesta III, Q. Song, D. R. Williams, C. W. Rowley, and F. S. Alvi, "Active control of flow-induced cavity oscillations," Progress in Aerospace Sciences, vol. 44, no. 7-8, pp. 479-502, 2008.

[6] S. J. Lawson and G. N. Barakos, "Review of numerical simulations for high-speed, turbulent cavity flows," Progress in Aerospace Sciences, vol. 47, no. 3, pp. 186-216, 2011.

[7] D. R. Williams, D. Cornelius, and C. W. Rowley, "Supersonic cavity response to open-loop forcing," Notes on Numerical Fluid Mechanics and Multidisciplinary Design, vol. 95, pp. 230-243, 2007.

[8] N. S. Vikramaditya and J. Kurian, "Pressure oscillations from cavities with ramp," AIAA Journal, vol. 47, no. 12, pp. 2974-2984, 2009.

[9] A. D. Vakili and C. Gauthier, "Control of cavity flow by upstream mass-injection," Journal of Aircraft, vol. 31, no. 1, pp. 169-174, 1994.

[10] D. P. Rizzetta and M. R. Visbal, "Large-eddy simulation of supersonic cavity flowfields including flow control," AIAA Journal, vol. 41, no. 8, pp. 1452-1462, 2003.

[11] L. Ukeiley, M. Sheehan, F. Coiffet, F. Alvi, S. Arunajatesan, and B. Jansen, "Control of pressure loads in geometrically complex cavities," Journal of Aircraft, vol. 45, no. 3, pp. 1014-1024, 2008.

[12] S. Arunajatesan, C. Kannepalli, N. Sinha et al., "Suppression of cavity loads using leading-edge blowing," AIAA Journal, vol. 47, no. 5, pp. 1132-1144, 2009. 
[13] T. Lusk, L. Cattafesta, and L. Ukeiley, "Leading edge slot blowing on an open cavity in supersonic flow," Experiments in Fluids, vol. 53, no. 1, pp. 187-199, 2012.

[14] W. Li, T. Nonomura, and K. Fujii, "Mechanism of controlling supersonic cavity oscillations using upstream mass injection," Physics of Fluids, vol. 25, no. 8, Article ID 086101, 2013.

[15] W. Li, T. Nonomura, and K. Fujii, "On the feedback mechanism in supersonic cavity flows," Physics of Fluids, vol. 25, no. 5, Article ID 056101, 2013.

[16] L. S. Ukeiley, M. Sheehan, F. Coiffet, F. S. Alvi, S. Arunajatesan, and B. Jansen, "Control of pressure loads in geometrically complex cavities," Journal of Aircraft, vol. 45, no. 3, pp. 10141024, 2008.

[17] W. Li, T. Nonomura, A. Oyama, and K. Fujii, "Feedback mechanism in supersonic laminar cavity flows," AIAA Journal, vol. 51, no. 1, pp. 253-257, 2013.

[18] D. Rockwell and C. Knisely, "The organized nature of flow impingement upon a corner," Journal of Fluid Mechanics, vol. 93, no. 3, pp. 413-432, 1979. 


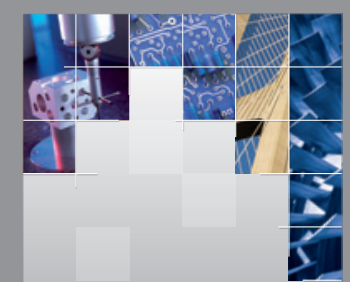

\section{Enfincering}
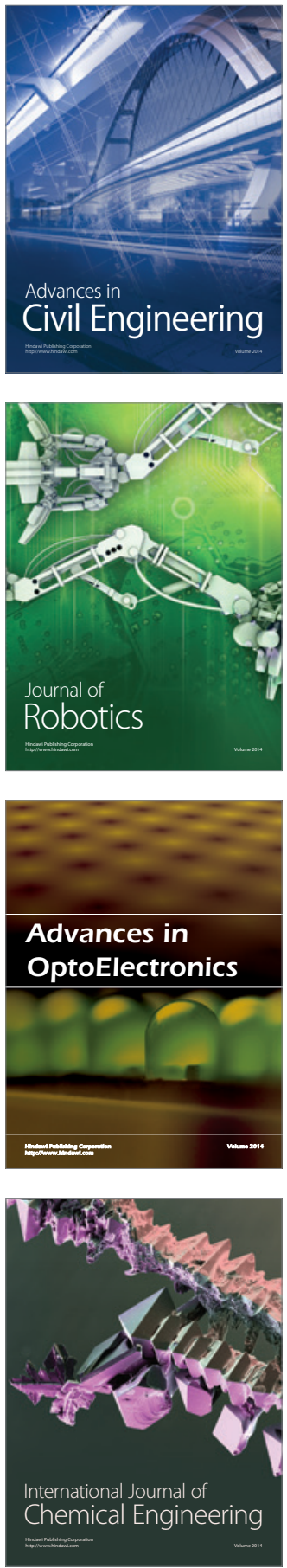

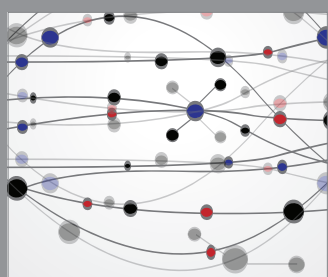

The Scientific World Journal

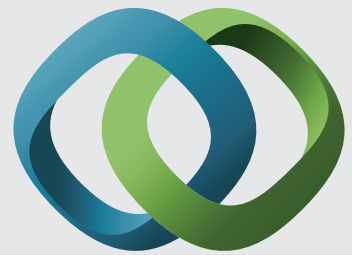

\section{Hindawi}

Submit your manuscripts at

http://www.hindawi.com
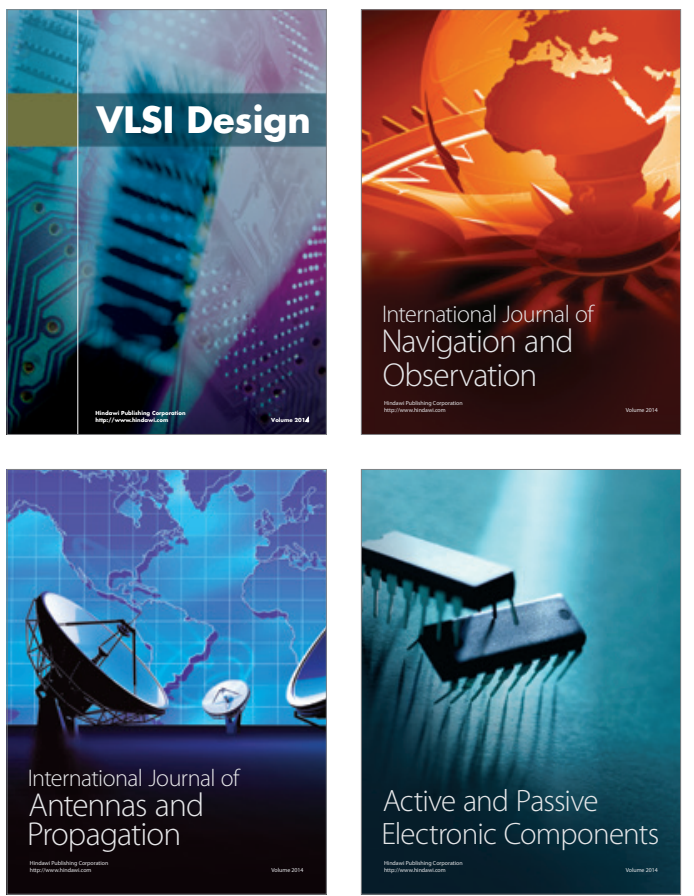
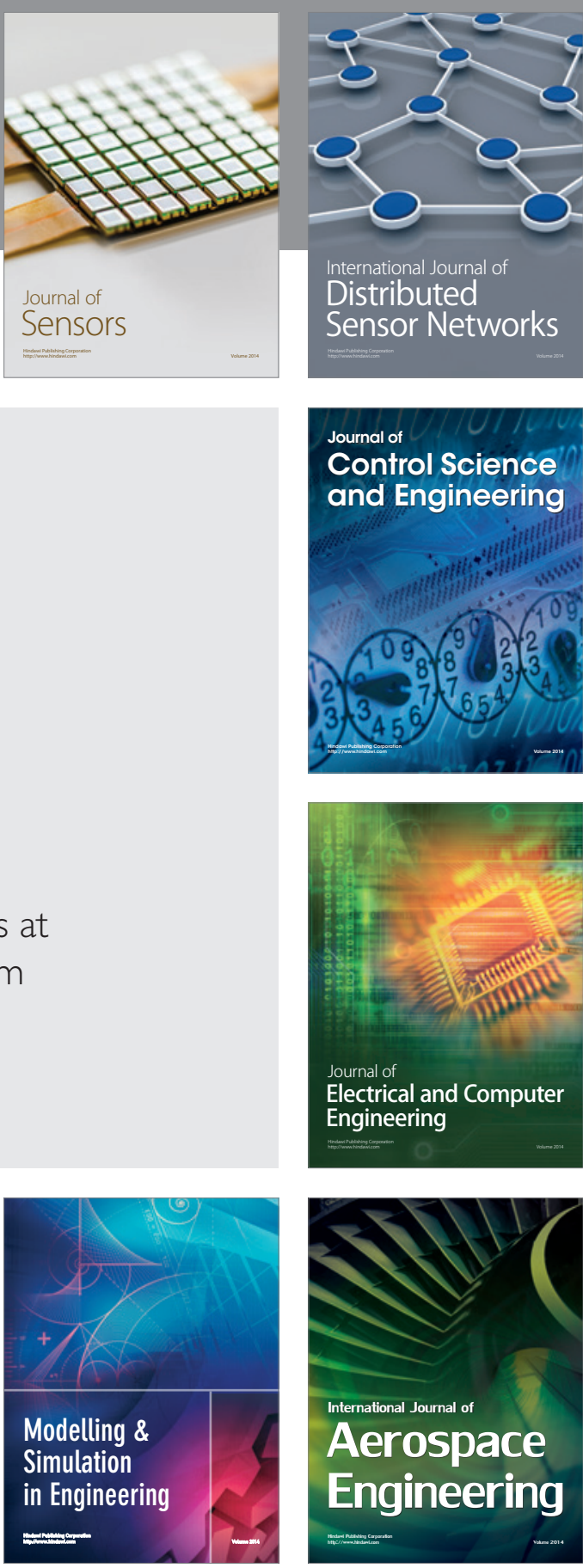

International Journal of

Distributed

Sensor Networks

Journal of

Control Science

and Engineering
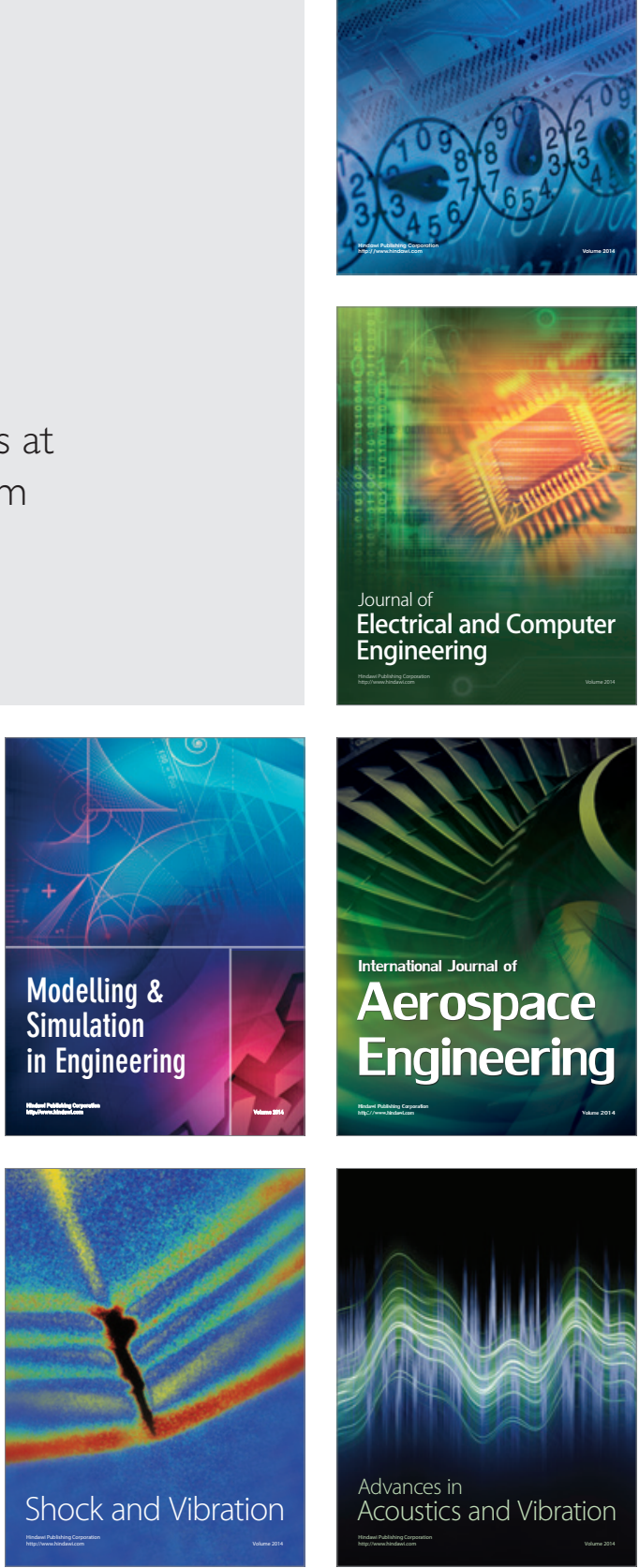